\title{
Development of CFTR structure
}

\author{
Anna E. Patrick and Philip J. Thomas* \\ Department of Physiology, University of Texas Southwestern Medical Center, Dallas, TX, USA
}

\section{Edited by:}

Marc Chanson, University of Geneva, Switzerland

\section{Reviewed by:}

Isabelle Callebaut, Centre National de la Recherche Scientifique, France Mohamed Benharouga,

CEA-CNRS-University of Joseph

Fourier, France

\section{*Correspondence}

Philip J. Thomas, Department of Physiology, University of Texas

Southwestern Medical Center, 5323 Harry Hines Boulevard, Dallas, TX

75390-9040, USA.

e-mail:philip.thomas@

utsouthwestern.edu
Cystic fibrosis is a lethal genetic disease caused by lack of functional cystic fibrosis transmembrane conductance regulator (CFTR) proteins at the apical surface of secretory epithelia. CFTR is a multidomain protein, containing five domains, and its functional structure is attained in a hierarchical folding process. Most CF-causing mutations in CFTR, including the most common mutation, a deletion of phenylalanine at position $508(\Delta \mathrm{F} 508)$, are unable to properly fold into this functional native three dimensional structure. Currently, no highresolution structural information about full length CFTR exists. However, insight has been gained through examining homologous $\mathrm{ABC}$ transporter structures, molecular modeling, and high-resolution structures of individual, isolated CFTR domains. Taken together, these studies indicate that the prevalent $\Delta \mathrm{F} 508$ mutation disrupts two essential steps during the development of the native structure: folding of the first nucleotide binding domain (NBD1) and its later association with the fourth intracellular loop (ICL4) in the second transmembrane domain (TMD2). Therapeutics to rescue $\triangle \mathrm{F} 508$ and other mutants in CFTR can be targeted to correct defects that occur during the complex folding process. This article reviews the structural relationships between CFTR and $A B C$ transporters and current knowledge about how CFTR attains its structure-with a focus on how this process is altered by CF-causing mutations in a manner targetable by therapeutics.

Keywords: CFTR, cystic fibrosis, ABC transporter, membrane protein structure, multidomain protein folding

\section{INTRODUCTION}

Cystic fibrosis (CF) is an autosomal recessive disease affecting more than 70,000 people world-wide. CF is caused by mutations in the gene encoding the CF transmembrane conductance regulator (CFTR) protein (Kerem et al., 1989; Riordan et al., 1989; Rommens et al., 1989). CFTR functions as a regulated chloride channel in the apical membrane of epithelia, where it plays a critical role in maintaining the surface liquid layer. Lack of functional CFTR results in thick secretions that cause gastrointestinal, reproductive, and respiratory system defects. Currently, CF patients most commonly die of respiratory-associated problems.

More than $70 \%$ of $\mathrm{CF}$ patients have at least one allele with a deletion of phenylalanine at position $508(\Delta$ F508; Kerem et al., 1989). Further sequencing of CF patient and non-patient CFTR genes has been extensive, and hundreds of mutations have been identified $^{1}$. Many of these mutations have been validated as CFcausing, while others are CF-associated but unstudied. The validated CF-causing mutations are located throughout the CFTR gene, and are inherited in almost all cases (Riordan et al., 1989; Riordan, 2008). $\Delta$ F508 (Cheng et al., 1990; Thomas et al., 1992) and many other CF mutations (Gregory et al., 1991) result in mutant CFTR that does not properly fold and is retained in the ER by cell protein quality control. The result is that more than $90 \%$ of mutant CFTR alleles produce a misfolded protein that is recognized, mistrafficked, and degraded in the cell.

While we do not have high-resolution three dimensional structural information for full length CFTR, a great deal of correlative

\footnotetext{
${ }^{1}$ www.genet.sickkids.on.ca
}

information regarding this structure has been obtained via homologous structures, domain structures, molecular modeling, and lower resolution techniques.

\section{ABC TRANSPORTERS}

Cystic fibrosis transmembrane conductance regulator is a member of the ATP-binding cassette (ABC) transporter superfamily of proteins, which includes membrane spanning proteins that use nucleotide hydrolysis to transport substrates across the membrane bilayer (Holland, 2003). While there is no full length highresolution structure for CFTR, there are structures for other ABC transporters, providing insight into the structure arrangement and functional mechanisms of CFTR. Most ABC transporters function to move substrates either into the cytoplasm (importers) or out of the cytoplasm (exporters). Exporters are found in both eukaryotes and prokaryotes, while importers have only been found in prokaryotes (Rees et al., 2009). The importance of prokaryotic ABC transporters for cellular functions, such as import of nutrients and export of toxins, is highlighted by their representation as 5\% of the Escherichia coli genome (Linton and Higgins, 1998). In humans, 48 or 49 distinct $A B C$ transporters have been identified, many of which are implicated in disease (Dean et al., 2001; Gottesman and Ambudkar, 2001; Borst and Elferink, 2002). The core $\mathrm{ABC}$ transporter architecture is comprised of two transmembrane spanning domains (TMDs) and two nucleotide binding domains (NBDs). Many transporters also have accessory domains with regulatory functions (Biemans-Oldehinkel et al., 2006). In general, the TMDs are organized as two wings that open and close in response to NBD movements resulting from ATP binding and 
hydrolysis (Figure 1; Moody et al., 2002; Smith et al., 2002; Locher, 2009; Rees et al., 2009). Additionally, at the external surface, many prokaryotic importers interact with accessory proteins that play a role in substrate transport (Biemans-Oldehinkel et al., 2006). The domains are modular, and are found expressed individually, in combinations, or as a single full length transporter to form the functional protein (Locher, 2009).

ATP-binding cassette transporters have a conserved coupling mechanism, whereby signals from the NBDs are transmitted to the intracellular loops (ICLs) of TMDs to cause substrate transport (Locher, 2009). The conserved NBDs form a sandwich around two ATPs, with each site for ATP binding and hydrolysis requiring both domains (Smith et al., 2002). Two subdomains are present in each NBD. The catalytic subdomain contains the conserved Walker A and B motifs, a Q-loop, and an H-motif, and the alpha-helical subdomain contains the ABC signature motif, LSGGQ (Figure 1C; Rees et al., 2009). Each active site is composed of components from the catalytic subunit of one NBD and the alpha-helical components of the other NBD in a head-to-tail arrangement (Smith et al., 2002; Rees et al., 2009). The binding of ATP in these sites drives the association of the NBDs (Moody et al., 2002).

The TMDs are proposed to function in an alternating access model of transport and are the most variable among ABC transporters (Chen et al., 2001; Dawson et al., 2007). ABC transporters can be divided into three classes based on the TMD fold (Locher, 2009). Type I and II ABC importers contain different core transmembrane (TM) span topologies of 10 and 20 TM helices respectively, with the latter tending to facilitate transport of larger substrates (Locher et al., 2002; Hollenstein et al., 2007; Locher, 2009). In both importer types, one TMD interacts with one NBD to form two TMD-NBD units that together form a functional transporter (Figure 1A; Locher, 2009). ABC exporters contain a core of 12 TM helices, with each wing of the transporter made of both TMDs, with each TMD interacting with both NBDs in a domain-swapped fashion (Figure 1B; Dawson and Locher, 2006; Locher, 2009). In this arrangement, the ICLs extend into the cytoplasm, positioning the NBDs approximately $25 \AA$ from the membrane (Figure 1B; Locher, 2009). In exporters, the TMDs and NBDs are expressed as TMD-NBD units, and eukaryotic exporters are most frequently found as full length transporters (Nikles and Tampe, 2007).

\section{CFTR AS AN ABC TRANSPORTER}

Cystic fibrosis transmembrane conductance regulator is a member of the ABC C subfamily, and is structurally homologous to the domain-swapped exporters. Structures of homologous ABC exporters such as bacterial Sav1866 (Dawson and Locher, 2006, 2007), bacterial MsbA (Ward et al., 2007), bacterial TM287/288 (Hohl et al., 2012), and mammalian P-glycoprotein (Aller et al., 2009) have been solved. The available structural data in combination with sequence alignments form the basis for homology models of full length CFTR that provide insight into its structure, mechanisms of regulation, and signal transduction (Mendoza and Thomas, 2007; Mornon et al., 2008, 2009; Serohijos et al., 2008). The exporter structures are in both open and closed forms, giving insight into movements within the CFTR protein during a transport cycle (Figure 2, open form; Ward et al., 2007; Locher, 2009; Mornon et al., 2009; Rees et al., 2009). The similarity of CFTR movements to other ABC transporters is supported by electron microscopy data in combination with a low resolution crystal structure (Rosenberg et al., 2004, 2011; Zhang et al., 2009,

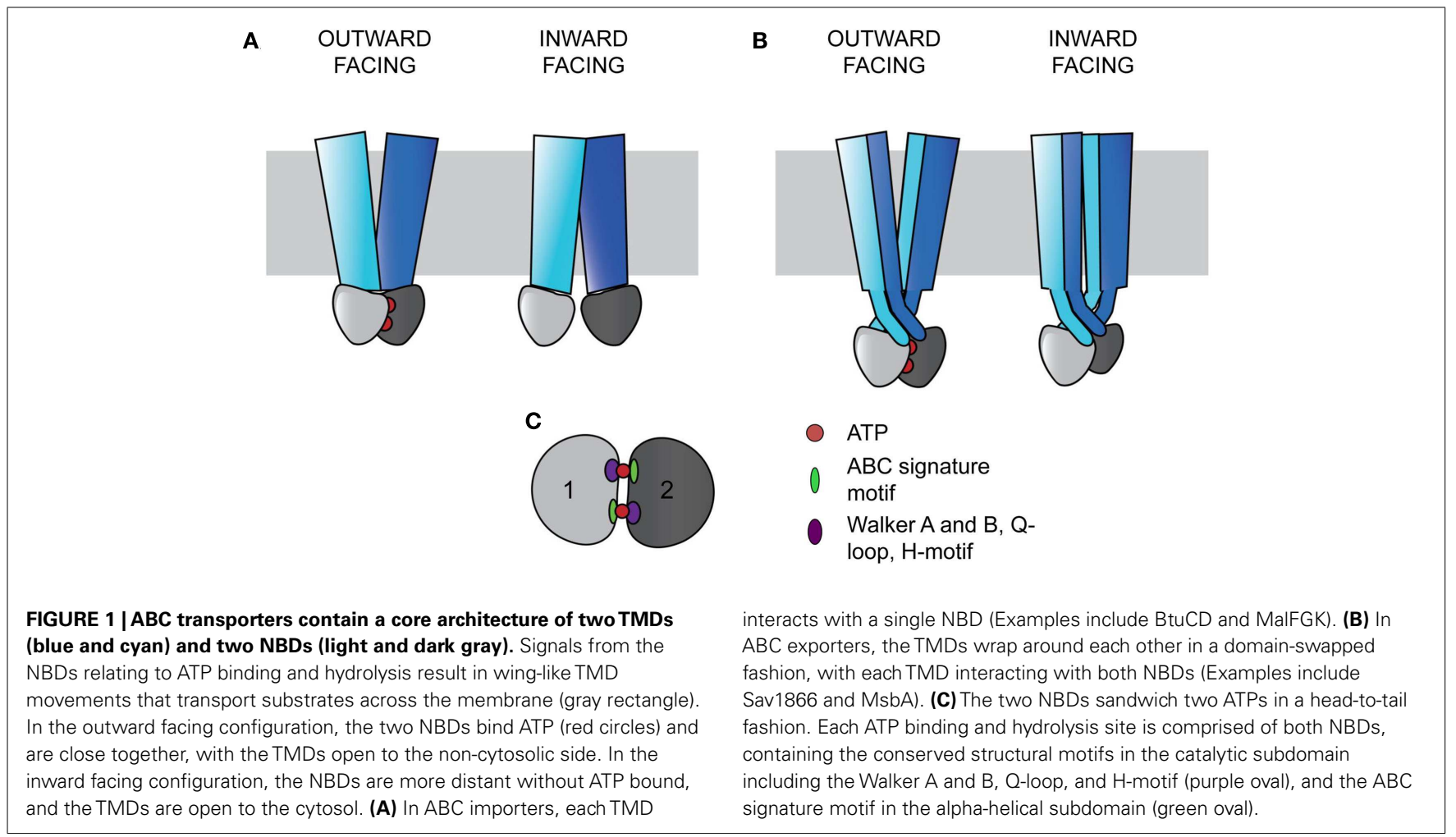



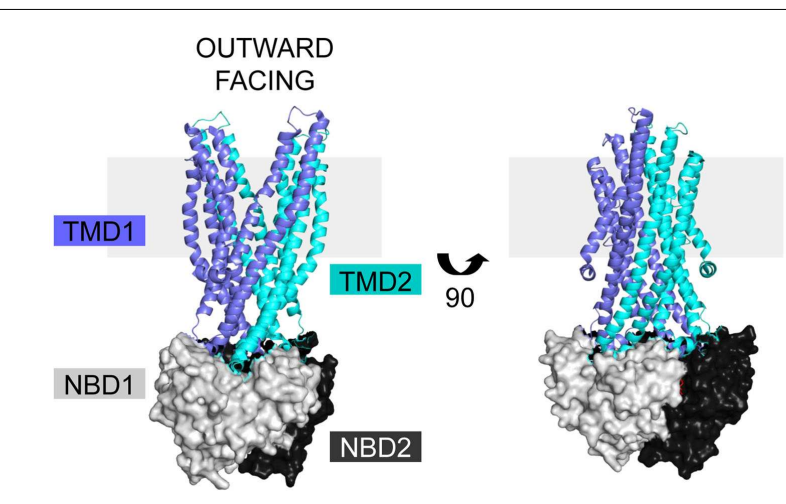

FIGURE 2 | CFTR homology models are based on the ABC exporter structures. The Sav1866 (pdb 2HYD) exporter is shown with domains colored based on representative CFTR domains, with TMD1 (blue), NBD1 (light gray), TMD2 (cyan), and NBD2 (black). There is no homologous structure for the CFTR R domain. The structure is in the outward facing configuration, which for CFTR is the open channel. The view is shown in the plane of the membrane (gray rectangle).

2011). The only high-resolution structures of CFTR domains are of NBD1 (Lewis et al., 2004, 2005, 2010; Thibodeau et al., 2005) and NBD2 (pdb 3GD7). As an ABC transporter, CFTR contains two TMDs, two NBDs, and a unique regulatory $\mathrm{R}$ region translated from an mRNA transcript as a single polypeptide chain (Riordan et al., 1989). Sav1866 based CFTR models have extensive interdomain interactions between the TMDs and NBDs, but lack regions without sequence homology, like the $\mathrm{R}$ domain (Figure 2; Dawson and Locher, 2006; Mendoza and Thomas, 2007).

The NBDs of CFTR, like other ABC transporters (Moody et al., 2002; Smith et al., 2002), interact in a head-to-tail fashion forming two sandwiched ATP binding pockets made of both domains (Vergani et al., 2005; Mense et al., 2006). Each NBD has a catalytic subdomain that contains the Walker A and B motifs and an alpha-helical subdomain that contains the conserved ABC signature motif (Lewis et al., 2004; Thibodeau et al., 2005). However, like several other members of the ABC C subfamily, one ATP binding site is non-hydrolytic (Muallem and Vergani, 2009). In this site, non-conservative mutations, which are located in the NBD1 Walker B and switch motifs and in the NBD2 signature sequence, result in tight binding and inefficient ATP hydrolysis (Aleksandrov et al., 2002; Basso et al., 2003; Gadsby et al., 2006). In general, CFTR ATP driven conformational changes include ATP binding, which results in an NBD dimer that signals the TMDs to open. Then, hydrolysis of one ATP disrupts the NBD interface, the NBDs separate, and the channel closes (Gadsby et al., 2006; Aleksandrov et al., 2007; Muallem and Vergani, 2009). However, the driving forces that control the gating transitions and the signals transmitted by ATP binding and hydrolysis are a matter of debate (Gadsby et al., 2006; Aleksandrov et al., 2007; Muallem and Vergani, 2009). NBD1 also contains two non-conserved regions, a regulatory insert (RI) near the $\mathrm{N}$-terminus and a regulatory extension (RE) near the C-terminus. Of these two regions, studies have focused on the RI. The RI is disordered in the NBD1 crystal structures and plays a role in regulation of CFTR channel gating, but is not required for trafficking in the cell (Lewis et al., 2004; Thibodeau et al., 2005; Aleksandrov et al., 2010). Furthermore, a mechanism wherein RI movements alter ICL1-NBD1 interactions to affect phosphorylation-dependent CFTR gating has been proposed (Kanelis et al., 2010).

Like other domain-swapped exporters, the TMDs form two wings containing TMs from both TMD1 and TMD2, such that the first two TMs and last four TMs of each domain make a wing (Figure 2; Dawson and Locher, 2006; Mendoza and Thomas, 2007). Based on the exporter structures, the wings move to open and close the chloride channel for ion transport (Vergani et al., 2005; Mornon et al., 2009). In each TMD, two alpha-helical ICLs extend into the cytoplasm, with each having a distal coupling helix that interacts with the NBDs (Mendoza and Thomas, 2007; Mornon et al., 2008; Serohijos et al., 2008). In combination, the four ICLs form four helix inner and outer bundles that end in the coupling helices (Figure 2; Mornon et al., 2008, 2009). Each coupling helix is parallel to the NBD surface, and forms a largely hydrophobic interface (Mendoza and Thomas, 2007). In CFTR, ICL2 interacts with NBD2, ICL4 interacts with NBD1, and ICLs 1 and 3 interact with both NBD1 and NBD2. Importantly, the F508 position in NBD1 is predicted to lie near the interface between NBD1 and ICL4 (Mendoza and Thomas, 2007). Many of the predicted interdomain interactions are also experimentally validated by crosslinking studies (Chen et al., 2004; Mense et al., 2006; He et al., 2008; Loo and Clarke, 2008; Serohijos et al., 2008). Further complexity of the ICL-NBD interactions is generated by phosphorylation-dependent interactions between NBD1 and an ICL1 peptide (Kanelis et al., 2010). Additionally, crosslinks between an ICL and the opposing NBD disrupt channel opening, supporting the essential roles of these components for channel function (He et al., 2008). Models predict specific residues are critical for the interactions between the ICLs and NBDs, including Y275 and W277 which form an interface with NBD2 (He et al., 2008; Mornon et al., 2008); D173, S169, and R170 which are predicted to contact nucleotide and NBD1 (Mornon et al., 2008); and S263 and E267 which stabilize ICL helical bundle structure (Mornon et al., 2009). Notably, the W277 position is equivalent to the R1070 position in ICL4 (Mornon et al., 2008) that when mutated, R1070W, suppresses the $\Delta$ F508 mutation (Thibodeau et al., 2010; Mendoza et al., 2012). These positions have not yet been fully tested for their roles in the folding and function of CFTR.

In summary, conformational signals generated in the NBDs in relation to ATP binding and hydrolysis are transmitted by the ICLs in the TMDs, resulting in chloride channel opening and closing (Gadsby et al., 2006; Riordan, 2008). The interactions between CFTR ICLs and NBDs have been validated by crosslinking studies (He et al., 2008; Serohijos et al., 2008) and complementation of a mutant located in an NBD with a mutant in an ICL (Thibodeau et al., 2010). The coupling helices of ABC transporters are architecturally conserved without having a highly conserved sequence (Locher, 2009), making prediction of essential positions and residues difficult without a high-resolution full length CFTR structure. 
Cystic fibrosis transmembrane conductance regulator is the only known channel among the $\mathrm{ABC}$ transporters. In the alternating access model, $\mathrm{ABC}$ transporters are open to one side of the membrane bilayer at a time (Chen et al., 2001; Dawson et al., 2007). In CFTR, channel formation abrogates this model, as one of the gates that would normally block substrate transport must be atrophied or gone to allow chloride flux (Gadsby, 2009). With regard to this, CFTR has been called a broken ABC transporter (Jordan et al., 2008; Muallem and Vergani, 2009). Similar to other chloride channels, CFTR is not very selective among small monovalent anions and has a relatively featureless pore (Gadsby et al., 2006; Gadsby, 2009). Putative residues that make the chloride channel have been identified in TMs and in extracellular loops, with a focus on TM1 and TM6 (Linsdell, 2006). However, it is difficult to validate these residues without better characterizing the TM span positions and TMD structures. Further complicating the TMD structure is a TMD1 N-terminal cytosolic region that regulates CFTR channel activity through interactions with the $\mathrm{R}$ domain, neither of which has a homologous structure (Naren et al., 1999; Chappe et al., 2005).

The chloride channel activity of CFTR is regulated by the R domain (Riordan, 2008). The $\mathrm{R}$ domain is largely unstructured and has multiple sites that are phosphorylated by PKA, resulting in CFTR channel activation (Gadsby et al., 2006; Baker et al., 2007). Consistent with this, the unphosphorylated $\mathrm{R}$ domain has an inhibitory effect on the CFTR channel (Rich et al., 1991; Csanady et al., 2000). The $\mathrm{R}$ domain interacts with multiple other regions of CFTR, including NBD1 and the N-terminus of TMD1 (Naren et al., 1999; Baker et al., 2007; Kanelis et al., 2010). This evidence suggests the $\mathrm{R}$ domain may act as a signal integrator to regulate channel function via interactions with different regions of CFTR. However, due to its lack of homology and disordered nature, the $\mathrm{R}$ domain location within CFTR models remains unclear.

Many different modifications to the CFTR protein that may impact its structure have been identified. For instance, CFTR contains two $N$-linked glycosylation sites, NXS/T $(\mathrm{X} \neq \mathrm{P})$, within TMD2 that are core glycosylated in the ER lumen. This core glycosylation is then modified in the Golgi to produce complex glycosylated protein (Helenius and Aebi, 2001). The natural sites within CFTR are regularly used to monitor its integration and cellular trafficking by changes in electrophoretic mobility upon core glycosylation, producing Band $\mathrm{B}$ at approximately $150 \mathrm{kDa}$, and complex glycosylation, producing a diffuse Band C above $170 \mathrm{kDa}$. The natural glycosylation sites are not required for cellular trafficking from the ER and chloride channel function (Howard et al., 1995; Chang et al., 2008; Glozman et al., 2009; Patrick et al., 2011). However, recently, these sites have been found to influence the efficiency of CFTR productive protein folding and early secretory trafficking (Glozman et al., 2009), and cell surface retention and turnover in post-ER cellular compartments (Chang et al., 2008; Glozman et al., 2009). The impact of these and other modifications on the development of CFTR structure is an area of ongoing study.

The combination of experimental and modeling studies provides significant insight into the CFTR structure, which allows formation of models within which mechanochemical mechanisms and the effect of CF-causing folding mutations can be framed.
However, since many CF-causing mutations, including $\Delta \mathrm{F} 508$, result in misfolding of the CFTR protein, the folded full length structure may not adequately describe the relevant defects.

\section{CFTR FOLDING AS A MULTIDOMAIN PROTEIN}

Cystic fibrosis transmembrane conductance regulator, like other $\mathrm{ABC}$ transporters, contains extensive interdomain surfaces (Rees et al., 2009) that, in the case of CFTR, likely form during translation (Zhang et al., 1998; Du et al., 2005; Kleizen et al., 2005; Thibodeau et al., 2005). During protein translation, secondary structure can begin to form early, even while the nascent chain is in the tunnel of the ribosome (Kramer et al., 2001; Woolhead et al., 2004). For CFTR, as translation continues each domain folds and can then interact with previously translated domains to form multidomain folding intermediates (Figure 3; Lukacs et al., 1994; Du et al., 2005; Kleizen et al., 2005; Thibodeau et al., 2005; Cui et al., 2007; Cheung and Deber, 2008; Du and Lukacs, 2009). The current model of CFTR folding holds that individual domain structures form cotranslationally (Kleizen et al., 2005). Then, intermediate structures form and eventually a TMD1-NBD1-R-TMD2 structure is produced that is required for cellular trafficking (Meacham et al., 1999; Du et al., 2005; Cui et al., 2007; Du and Lukacs, 2009). Finally, NBD2 posttranslationally incorporates into the CFTR structure (Figure 3; Du et al., 2005). The addition of NBD2 confers a greater folding efficiency and trafficking from the ER. Thus, although NBD2 is not strictly required for CFTR trafficking (Pollet et al., 2000; Cui et al., 2007; Du and Lukacs, 2009; Thibodeau et al., 2010), its posttranslational association into the CFTR structure (Du et al., 2005) may increase the yield of folded cellular CFTR. Much of this model is based on individual CFTR domains forming protease-resistant structures during translation (Zhang et al., 1998; Kleizen et al., 2005). The order of interdomain interaction formation and whether initial interactions are the same as those in the final CFTR structure is not known.

Cystic fibrosis transmembrane conductance regulator folding occurs during translation as a linear polypeptide (Riordan et al., 1989). However, many ABC transporter domains are expressed separately, and later associate to form a functional transporter (Locher, 2009). To some extent, CFTR retains some ability to fold in this manner. CFTR can be expressed as a split construct, which forms structure that traffics to the cell surface and functions as a chloride channel (Ostedgaard et al., 1997; Chan et al., 2000; Csanady et al., 2000; Du and Lukacs, 2009). Additionally, expression of constructs containing TMD1-NBD1-R or R-TMD2-NBD2 formed chloride channels, likely as multimers (Sheppard et al., 1994; Devidas et al., 1998). Finally, in the cell the minimal construct that traffics from the ER contains TMD1-NBD1-R-TMD2, which forms a chloride channel (Cui et al., 2007). These studies suggest that the domains of CFTR, to a certain extent, can associate posttranslationally to form a functional chloride channel. Yet, CFTR is a linear chain, such that folding requires that each domain attain structure in a more spatially confined manner. The critical role of the primary sequence in the CFTR folding process is highlighted by the multitude of CF-associated folding mutations identified throughout the protein (see text footnote 1). 


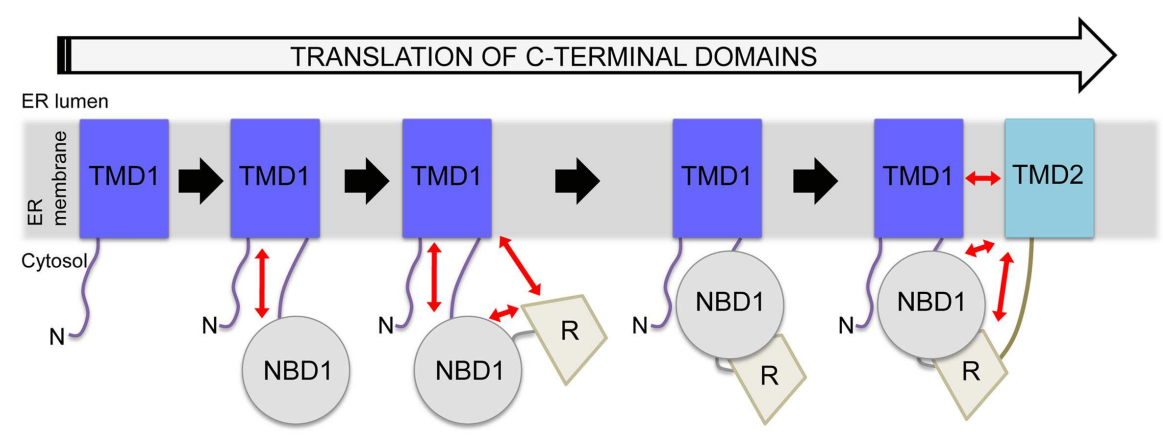

TRANSLATION OF C-TERMINAL DOMAINS

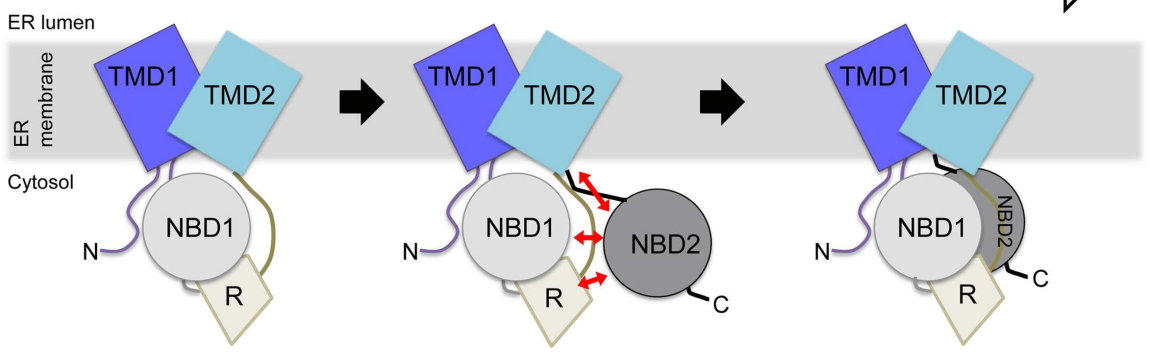

FIGURE 3 | Hierarchical folding model of CFTR. Potential interdomain interactions are indicated by red arrows, with several possible structural units included during the translation process. In the cell, constructs lacking TMD2 do not traffic from the ER, whereas constructs containing TMD2 can traffic from the ER. Reflecting this, a major structural rearrangement is depicted in the presence of TMD2, as shown in the bottom panel. Eventually, NBD2 is incorporated as the final step. There are many points during this process at which the cell may monitor perturbations.

\section{CFTR COTRANSLATIONAL FOLDING INVOLVES INTERACTIONS WITH OTHER PROTEINS}

Cystic fibrosis transmembrane conductance regulator folding involves many proteins that act at different stages to aid folding or recognize misfolding. This topic is extensively reviewed elsewhere within this Research Topic. Briefly, the misfolded CFTR is retained in the ER, and eventually degraded (Lukacs et al., 1994) by the proteasome (Jensen et al., 1995; Ward et al., 1995). Many proteins interact with CFTR in the ER lumen, ER membrane, and cytoplasm, suggesting that the domains of CFTR are differentially monitored during the biosynthetic process. Among these identified interacting partners are the cytoplasmic proteins Hsc/p 40, 70, 90, and associated co-chaperones CHIP (Strickland et al., 1997; Meacham et al., 1999, 2001; Younger et al., 2006) and Ahal (Wang et al., 2006), the ER membrane associated protein RMA1 (Younger et al., 2006; Grove et al., 2011), the ER integral membrane proteins Derlin (Sun et al., 2006; Younger et al., 2006; Wang et al., 2008) and BAP31 (Wang et al., 2008), and the ER luminal-interacting protein calnexin (Pind et al., 1994). After trafficking to the cell surface, CFTR interactions with cytoskeletal proteins are important for its maintenance at this cellular location (Okiyoneda and Lukacs, 2007). Also at the plasma membrane, peripheral protein quality control is involved in the ubiquitination, internalization, and degradation of misfolded CFTR (Okiyoneda et al., 2010). Moreover, a protein interactome for CFTR includes potential interactions far beyond those that have been studied (Wang et al., 2006). However, it is not clear which proteins interact at the earliest stages of folding/maturation and are responsible for initial and irreversible recognition of mutant CFTR. Furthermore, the structural aspects of CFTR during folding that are important for formation of these interactions are unclear. These interactions paint a picture of CFTR biogenesis whereby normal structural formation and interactions are formed with cellular folding and quality control machinery, providing multiple points to monitor CFTR folding.

\section{CF-MUTANTS PERTURB CFTR COTRANSLATIONAL FOLDING}

Cystic fibrosis-associated mutations have been found in every domain of CFTR (see text footnote 1). Misfolded CFTR, specifically the $\Delta$ F508 mutant protein, is recognized by cellular quality control machinery, accumulates in the ER (Cheng et al., 1990), and is eventually degraded (Lukacs et al., 1994) by the proteasome (Jensen et al., 1995; Ward et al., 1995). Many studies have identified CF-causing mutants that result in accumulation of CFTR in the ER. Mutant effects have been categorized into classes based on the resulting effect on CFTR (Welsh and Smith, 1993; Zielenski and Tsui, 1995). The alterations include lack of protein production (class I), defective protein maturation and early degradation (class II), defective regulation of ATP interactions (class III), reduced chloride transport (class IV), reduced transcripts though splicing or promoter defects (class V), and increased cell surface turnover (class VI; Welsh and Smith, 1993; Zielenski and Tsui, 1995). The $\Delta$ F508 mutation accounts for $70 \%$ of CF-causing mutant CFTR alleles (Riordan et al., 1989), making class II defects the most common cause of CF. 
Mutations within the CFTR protein, including $\Delta$ F508, may perturb local protein structure and/or domain structure, or could be surface exposed and perturb interactions with other domains or proteins. For instance, in the NBD-ICL4 interface, mutants in ICL4 including L1065P, R1066C, and A1067T alter trafficking and chloride channel function (Cotten et al., 1996; Seibert et al., 1996). Mutants in different domains alter biogenic intermediates of CFTR, suggesting that misfolding does not require full length CFTR (Du and Lukacs, 2009). Furthermore, in full length CFTR, the proteolytic stability of all domains was reduced for spatially separate mutations, suggesting propagation of one mutant to other domains (Rosser et al., 2008; Du and Lukacs, 2009). The propagation of mutants could occur through a rearrangement step involving multiple domains ( $\mathrm{Du}$ and Lukacs, 2009), or through coupled folding of the domains. As discussed, various components of cell quality control recognize CFTR as it is created, such that domain and multidomain states are likely differentially monitored (Younger et al., 2006). For each mutation, the effect on individual domain folding and multidomain units plays a fundamental role in determining the mechanisms by which that mutation is recognized and managed within the cell.

An example of mutants similarly located within CFTR with different local mechanisms of misfolding are the G85E and G91R mutations. These mutations are located near or within the TM1 span within TMD1. Both mutations have been demonstrated to disrupt later steps in CFTR folding, including interdomain interactions, which have been proposed to result in mutant recognition by ER quality control machinery (Xiong et al., 1997). Recently, G85E was found to dramatically alter the conformation/integration profile of TM1 (Patrick et al., 2011). Such an alteration would occur at the earliest steps of translation and integration, and could be recognized as a very early misfolding event by ER quality control machinery. The G91R mutant was predicted to have a similar effect on CFTR (Xiong et al., 1997), but this proved not to be true with regards to the TM1 conformation/integration profile (Patrick et al., 2011). Interestingly, the corrector compound four rescues G91R but not G85E-CFTR (Grove et al., 2009), suggesting the differences in the mutant molecular pathologies may be relevant for their ability to benefit from specific treatments to rescue defective CFTR. The detailed mechanistic study of CF-causing mutations provides a better fundamental understanding of membrane protein misfolding and mechanisms for approaching mutant specific therapy for CF patients.

\section{F0LDING OF NBD1 AND $\triangle$ F508-NBD1}

The best studied disease-causing mutation, $\Delta F 508$, alters multiple steps during CFTR folding. Particular focus has been given to folding of NBD1, wherein F508 resides. High-resolution crystal structures of both NBD1 and $\triangle$ F508-NBD1 have been solved (Lewis et al., 2004, 2005; Thibodeau et al., 2005; Mendoza et al., 2012). These structures place F508 on the domain surface, and $\Delta$ F508 does not cause significant perturbations in the crystal structure (Lewis et al., 2005). However, $\Delta$ F508-NBD1 has an increased tendency to aggregate and is destabilized, indicating a disruption during folding that is not represented in these native structures (Qu and Thomas, 1996; Lewis et al., 2005; Thibodeau et al., 2005). Consistent with this, a non-native conformation of NBD1 has been identified that is promoted by $\Delta$ F508 and linked to increased aggregation (Hoelen et al., 2010; Richardson, unpublished data). The NBD1 structure is obtained cotranslationally (Kleizen et al., 2005; Hoelen et al., 2010; Khushoo et al., 2011). During translation, a ligand-dependent $\mathrm{N}$-terminal compact structure forms, and upon completion of NBD1 translation another compact structure forms (Khushoo et al., 2011). The compact N-terminal structure is not affected by $\Delta \mathrm{F} 508$, suggesting that the folding error likely occurs at a later step of NBD1 folding (Khushoo et al., 2011). The $\triangle$ F508 misfolding begins in NBD1, making this an attractive target for correcting $\Delta \mathrm{F} 508-\mathrm{CFTR}$. The $\Delta \mathrm{F} 508$ mutant effects can be partially rescued independently by suppressor mutations within NBD1 (Teem et al., 1993; Qu et al., 1997; DeCarvalho et al., 2002; Hoelen et al., 2010). Importantly, the $\Delta F 508$ effects on NBD1 also manifest during translation of the full length CFTR (Kleizen et al., 2005).

In full length CFTR, $\Delta$ F508 effects multidomain stability and interdomain interactions. In mammalian cells, the $\Delta$ F508-CFTR misfolds, resulting in cellular mistrafficking via its accumulation in the ER (Cheng et al., 1990). As shown by limited proteolysis and pulse chase analysis, the $\Delta$ F508 mutation destabilizes NBD1 and multidomain folding intermediates, implying a more global destabilization of the entire $\Delta$ F508-CFTR (Zhang et al., 1998; Meacham et al., 1999; Du et al., 2005; Cui et al., 2007; Rosser et al., 2008; Du and Lukacs, 2009). The homology model of CFTR places the F508 position at an interface between NBD1 and ICL4 of TMD2 (Mendoza and Thomas, 2007). Consistent with this, $\Delta$ F508 disrupts WT-like crosslinks between ICL4 and NBD1 and within the TMDs (Chen et al., 2004; Serohijos et al., 2008). Additionally, mutations in ICL4 can suppress the effect of $\Delta$ F508, further supporting a disruption of this interface (Thibodeau et al., 2010). Recently, the $\triangle$ F508-mediated NBD1 misfolding and multidomain assembly were both shown as essential for correction of $\Delta$ F508-CFTR (Mendoza et al., 2012; Rabeh et al., 2012). This is consistent with the known $\triangle \mathrm{F} 508$ effects on NBD1 folding, which is a prerequisite for its interdomain interactions and formation of an NBD1 surface for ICL4 interactions. However, these experiments have not yet been able to identify the timing or mechanism(s) of domain interaction disruption. The point at which $\Delta$ F508 effects are detectable and the ability to target multiple steps to rescue the $\Delta$ F508 protein emphasizes the multistep misfolding of $\Delta$ F508-CFTR. Further details regarding this misfolding are needed to continue to rationally devise new therapeutic interventions.

Other methods to rescue $\triangle$ F508-CFTR continue to be explored. For instance, compounds have been identified that rescue $\Delta$ F508-CFTR mutation via interactions with the TMDs (Loo et al., 2011). $\Delta$ F508 and other mutant CFTRs were also partially rescued by transcomplementation, in which co-expression of parts of CFTR were able to improve trafficking of CF-mutant CFTR from the ER (Cormet-Boyaka et al., 2004; Cebotaru et al., 2008). Insights into the rescue of $\Delta F 508$-CFTR also come from the yeast homologous ABC exporter, Yorlp (Pagant et al., 2007, 2008). When a $\Delta F 508$ mimic is introduced into Yorlp, consequent mistrafficking and degradation occurs (Pagant et al., 2007). Two Yorlp suppressor mutations in the TM-ICL juncture were found to correct the $\Delta$ F508 mimic (Pagant et al., 2010), suggesting that modification of the ICL structures rather than direct stabilization 
of the NBD-ICL interface is a potential target for correction of $\triangle$ F508-CFTR. Also, a co-expressed Yor1p NBD1 was able to swap into the $\Delta \mathrm{F} 508$ mimic-Yorlp to replace the defective domain (Louie et al., 2010). Notable differences exist between the Yor1p protein and CFTR; however these findings provide insight into potential mechanisms for $\triangle$ F508-CFTR correction that should be investigated directly with CFTR.

\section{RESCUING MUTANT CFTR}

It is suggested that only $10-35 \%$ of CFTR function is needed to positively impact pulmonary disease (Kerem, 2004), therefore the production and residual activity of mutant CFTR is relevant for clinical outcomes. In CF, there is a focus on rescuing the defective CFTR protein. Ongoing therapeutic developments are aimed at targeting mutations that introduce premature termination codons, decrease chloride channel function, and alter cellular trafficking, which are discussed elsewhere within this Research Topic. For $\Delta$ F508 and other missense mutations, two aspects to rescuing mutant CFTR protein are to rescue processing and function, both of which are innately linked to CFTR structure.

Thus far, great success has occurred in rescuing the CF-causing G551D mutant. G551D-CFTR has normal cell surface expression and half-life, but confers a severe defect in channel gating (Welsh and Smith, 1993). The compound VX-770 was initially characterized as a CFTR potentiator in CF airway epithelial cells (Van Goor et al., 2009). This compound has since undergone clinical trials showing efficacy in CF patients (Accurso et al., 2010; Ramsey et al., 2011), has been approved by the FDA for treatment of G551D based CF in patients over 6 years old, and is now marketed as Kalydeco ${ }^{\mathrm{TM}}$. These results are promising for CF patients as adults, who already have lung scarring and dysfunction, and for children, who may be able to avoid lung dysfunction with this therapeutic. This success has generated a foundation to guide further progress in CF therapeutic development for other mutants, such as $\Delta \mathrm{F} 508$.

$\triangle F 508$ and other mutants that cause CFTR misfolding, mistrafficking, and disrupted channel function are the largest $\mathrm{CF}$ therapeutic target. The $\triangle$ F508-CFTR exhibits a temperature sensitive trafficking from the ER, in which it is retained in the ER at $37^{\circ} \mathrm{C}$, but partially traffics from the ER at lower temperatures (Denning et al., 1992). This imparts the idea that trafficking correction is feasible for $\Delta \mathrm{F} 508$ if a chemical compound can mimic the temperature rescue. However, $\triangle F 508$-CFTR that is induced to fold/traffic by low temperature or chemical modifier treatments has disrupted chloride channel function (Dalemans et al., 1991) and shorter residence times at the cellular surface (Lukacs et al., 1993), indicating the native structure is not achieved. This makes approaching $\triangle$ F508-CFTR a complex problem. Recently, it was found that correction of both the $\triangle$ F508-NBD1 defect and the $\Delta$ F508-NBD1-ICL4 interaction defect are required to rescue $\Delta \mathrm{F} 508$, consistent with at least two steps for correction of $\Delta \mathrm{F} 508$ CFTR (Mendoza et al., 2012; Rabeh et al., 2012). $\Delta$ F508 is being targeted pharmacologically by strategies that aim to correct the trafficking defect and potentiate channel function. Currently trials of VX-809 or VX-661, to correct trafficking, and Kalydeco ${ }^{\mathrm{TM}}$, to potentiate channel function, are ongoing. However, development of a combination therapy is exponentially more complicated and difficult. Ideally, a single compound to both correct and potentiate mutant CFTR will be identified (Sheppard, 2011). Extensive work has gone into describing $\Delta$ F508-CFTR misfolding in order to identify the most pertinent misfolding $\operatorname{step}(\mathrm{s})$ for generating the most relevant therapeutic target.

\section{DISCUSSION}

Cystic fibrosis transmembrane conductance regulator structural development occurs in a complex manner (Figure 3). It requires formation of TMD1, which involves TM span interactions with the translocation machinery in the ER. Then production of two cytosolic domains occurs, first NBD1 and then R. Following this, yet another TMD must be appropriately integrated, with the protein structure completed after the production of cytosolic NBD2. In the final structure, these domains form extensive interdomain interactions, with the later interaction surfaces having no obvious interaction partners prior to formation of the final structure. For instance, during translation, the TM and ICL regions that form later interdomain interactions are present minutes prior to production of their interaction partners. These regions are very hydrophobic and are unlikely to be stable without their partner sequences or other protein interactions. While the ICL helical bundle likely forms only when both TMD1 and TMD2 are present, this has not been tested experimentally. It is not known if ICL structure formation begins in TMD1, or what happens to the coupling helices before both NBDs are present. A requirement of this structure for NBD docking onto the ICLs has not been examined. Knowledge of the timing of this structure formation and its role in TMD-NBD interactions will be required for better understanding development of $\mathrm{ABC}$ transporter structure. The interactions required for the formation of native CFTR structure are important for understanding CFmutant mediated misfolding, which is a therapeutic target for correcting CF-mutant CFTR.

Experimental evidence supports that the first four domains of CFTR undergo a multidomain rearrangement, since a regulated chloride channel that can traffic to the plasma membrane is formed (Cui et al., 2007). The cell is able to monitor and determine whether the TMD2 containing construct should traffic from the ER (Cui et al., 2007; Du and Lukacs, 2009; Thibodeau et al., 2010). This suggests that, upon the translation of TMD2, the protein quality control machinery makes a distinction between folded and unfolded CFTR. A hierarchical folding model also predicts that two and three domain hierarchical interactions also form (Figure 3). Though this model is appealing, little evidence exists to support domain associations prior to the translation of TMD2. The most suggestive evidence of interdomain interactions in the first two and three domains of CFTR is the formation of a more stable three domain construct (Meacham et al., 1999; Rosser et al., 2008; Grove et al., 2009). In these studies, the interdomain interactions are implied rather than directly tested. Much of the evidence for formation of multidomain units is forced to rely on the use of modeling and perturbing mutations to detect the structural units. It is clear, however, that the most highly studied mutants, specifically $\Delta \mathrm{F} 508$, alter domain structure in a manner recognizable by the cell (Du and Lukacs, 2009), convoluting the interpretation of multidomain complexes with domain effects. A continuing 
effort to analyze native and mutant CFTR and to develop assays to better study multidomain unit formation are required to continue addressing these specific issues.

It is important to consider that CFTR cotranslational interactions may be directly related to the order of domain translation. If these interactions are required sequentially for structure formation, then a linear peptide should be essential to produce folded CFTR. However, CFTR expressed as two pieces underwent cellular trafficking as monitored by glycosylation (Ostedgaard et al., 1997; Chan et al., 2000; Csanady et al., 2000; Du and Lukacs, 2009), inconsistent with the model. By contrast, the ability of split CFTR to form functional protein is consistent with other ABC transporters within which the modular formation of domain structure indicates that one domain is not required for the formation of other domains (Locher, 2009). Yet, during in vitro refolding of the modular $\mathrm{ABC}$ transporter, BtuCD, refolding from partially unfolded units resulted in the highest functional measures (Di Bartolo et al., 2011). This suggests that domain interactions during folding may play a role in increasing the production yield of functional protein. For CFTR, these interactions could be potentiated by the linear arrangement and be important for generating enough functional protein to maintain normal physiology. This may play a role in reaching a level of physiologically functional CFTR required to alter the progression of CF therapeutically.

Cystic fibrosis clinically impacts multiple organ systems, such that treatment of the basic defect in CFTR is the best way to address the widespread morbidities. Novel therapeutics show tremendous promise for altering the molecular pathologies of CF, however, implementation of therapeutics designed to correct the most common mutant, $\Delta \mathrm{F} 508$, is difficult. The $\Delta \mathrm{F} 508$ molecular pathology

\section{REFERENCES}

Accurso, F. J., Rowe, S. M., Clancy, J. P., Boyle, M. P., Dunitz, J. M., Durie, P. R., Sagel, S. D., Hornick, D. B., Konstan, M. W., Donaldson, S. H., Moss, R. B., Pilewski, J. M., Rubenstein, R. C., Uluer, A. Z., Aitken, M. L., Freedman, S. D., Rose, L. M., Mayer-Hamblett, N., Dong, Q., Zha, J., Stone, A. J., Olson, E. R., Ordonez, C. L., Campbell, P. W., Ashlock, M. A., and Ramsey, B. W. (2010). Effect of VX-770 in persons with cystic fibrosis and the G551DCFTR mutation. N. Engl. J. Med.363, 1991-2003.

Aleksandrov, A. A., Aleksandrov, L. A., and Riordan, J. R. (2007). CFTR (ABCC7) is a hydrolyzable-ligandgated channel. Pflugers Arch. 453, 693-702.

Aleksandrov, A. A., Kota, P., Aleksandrov, L. A., He, L., Jensen, T., Cui, L., Gentzsch, M., Dokholyan, N. V., and Riordan, J. R. (2010). Regulatory insertion removal restores maturation, stability, and function of deltaF508 CFTR. J. Mol. Biol. 401, 194-210.

Aleksandrov, L., Aleksandrov, A. A. Chang, X. B., and Riordan, J. R.

is complex and involves multiple levels of misfolding and recognition thereof in the cell. Indeed, $\Delta$ F508-CFTR misfolds and is accumulated in the ER (Cheng et al., 1990). Moreover, if the trafficking defect is overcome, cell surface $\Delta$ F508-CFTR displays reduced chloride transport (Dalemans et al., 1991) and an accelerated turnover rate (Lukacs et al., 1993). Addressing each effect individually is inadequate, and a successful combination of therapeutics has not yet been identified to effectively rescue the $\Delta \mathrm{F} 508$ mutation and remains an untraveled therapeutic path. Suppressor mutations of $\Delta \mathrm{F} 508$ have been identified within NBD1 (Teem et al., 1993) and within ICL4 (Thibodeau et al., 2010), which correct NBD1 folding and/or multidomain folding. But, individually, these suppressors have limited efficacy. It is now established that correction of at least two steps are needed to rescue $\Delta F 508$, including NBD1 folding and interdomain interactions (Mendoza et al., 2012; Rabeh et al., 2012). If the effects of the different suppressor mutations for $\triangle \mathrm{F} 508$ either within NBD1 or distant in the CFTR protein can be mimicked and combined in a small molecule this could prove an effective therapeutic. It is clear from these studies that the identification of disease mechanisms that may be targeted therapeutically requires a global understanding of CFTR structure. Future disease modifying compounds will be more effective if the target is the most relevant biological defect.

\section{ACKNOWLEDGMENTS}

This work was supported by grants from the National Institutes of Health (DK49835 and DE12309) and the Cystic Fibrosis Foundation (05XX0) to Philip J. Thomas and National Institutes of Health Ruth Kirschstein predoctoral fellowship F30 (DK087186) to Anna E. Patrick.

roles of accessory domains. FEBS Lett. 580, 1023-1035.

Borst, P., and Elferink, R. O. (2002). Mammalian $\mathrm{ABC}$ transporters in health and disease. Annu. Rev. Biochem. 71, 537-592.

Cebotaru, L., Vij, N., Ciobanu, I. Wright, J., Flotte, T., and Guggino, W. B. (2008). Cystic fibrosis transmembrane regulator missing the first four transmembrane segments increases wild type and deltaF508 processing. J. Biol. Chem. 283, 21926-21933.

Chan, K. W., Csanady, L., Seto-Young, D., Nairn, A. C., and Gadsby, D. C. (2000). Severed molecules functionally define the boundaries of the cystic fibrosis transmembrane conductance regulator's $\mathrm{NH}(2)$-terminal nucleotide binding domain. J. Gen Physiol. 116, 163-180.

Chang, X. B., Mengos, A., Hou, Y. X., Cui, L., Jensen, T. J., Aleksandrov, A., Riordan, J. R., and Gentzsch, M. (2008). Role of N-linked oligosaccharides in the biosynthetic processing of the cystic fibrosis membrane conductance regulator. J. Cell. Sci. 121, 2814-2823.

Chappe, V., Irvine, T., Liao, J., Evagelidis, A., and Hanrahan, J. W. (2005)
Phosphorylation of CFTR by PKA promotes binding of the regulatory domain. EMBO J. 24, 2730-2740.

Chen, E. Y., Bartlett, M. C., Loo, T. W., and Clarke, D. M. (2004). The deltaF508 mutation disrupts packing of the transmembrane segments of the cystic fibrosis transmembrane conductance regulator. J. Biol. Chem. 279, 39620-39627.

Chen, J., Sharma, S., Quiocho, F. A., and Davidson, A. L. (2001). Trapping the transition state of an ATPbinding cassette transporter: evidence for a concerted mechanism of maltose transport. Proc. Natl. Acad. Sci. U.S.A. 98, 1525-1530.

Cheng, S. H., Gregory, R. J., Marshall, J., Paul, S., Souza, D. W., White, G. A., O'riordan, C. R., and Smith, A. E. (1990). Defective intracellular transport and processing of CFTR is the molecular basis of most cystic fibrosis. Cell 63, 827-834. terminal nucleotide binding domain and its role in channel gating. J. Gen. Physiol. 122, 333-348.

Biemans-Oldehinkel, E., Doeven, M. K., and Poolman, B. (2006). ABC transporter architecture and regulatory
Cheung, J. C., and Deber, C. M. (2008). Misfolding of the cystic fibrosis transmembrane conductance regulator and disease. Biochemistry 47, 1465-1473. 
Cormet-Boyaka, E., Jablonsky, M., Naren, A. P., Jackson, P. L., Muccio, D. D., and Kirk, K. L. (2004). Rescuing cystic fibrosis transmembrane conductance regulator (CFTR)-processing mutants by transcomplementation. Proc. Natl. Acad. Sci. U.S.A. 101, 8221-8226.

Cotten, J. F., Ostedgaard, L. S., Carson, M. R., and Welsh, M. J. (1996). Effect of cystic fibrosis-associated mutations in the fourth intracellular loop of cystic fibrosis transmembrane conductance regulator. J. Biol. Chem. 271, 21279-21284.

Csanady, L., Chan, K. W., Seto-Young, D., Kopsco, D. C., Nairn, A. C., and Gadsby, D. C. (2000). Severed channels probe regulation of gating of cystic fibrosis transmembrane conductance regulator by its cytoplasmic domains. J. Gen. Physiol. 116, 477-500.

Cui, L., Aleksandrov, L., Chang, X. B., Hou, Y. X., He, L., Hegedus, T., Gentzsch, M., Aleksandrov, A., Balch, W. E., and Riordan, J. R. (2007). Domain interdependence in the biosynthetic assembly of CFTR. J. Mol. Biol. 365, 981-994.

Dalemans, W., Barbry, P., Champigny, G., Jallat, S., Dott, K., Dreyer, D., Crystal, R. G., Pavirani, A., Lecocq, J. P., and Lazdunski, M. (1991). Altered chloride ion channel kinetics associated with the delta F508 cystic fibrosis mutation. Nature 354 , 526-528.

Dawson, R. J., Hollenstein, K., and Locher, K. P. (2007). Uptake or extrusion: crystal structures of full $\mathrm{ABC}$ transporters suggest a common mechanism. Mol. Microbiol. 65, 250-257.

Dawson, R. J., and Locher, K. P. (2006). Structure of a bacterial multidrug ABC transporter. Nature 443, 180-185.

Dawson, R. J., and Locher, K. P. (2007). Structure of the multidrug $\mathrm{ABC}$ transporter Sav1866 from Staphylococcus aureus in complex with AMP-PNP. FEBS Lett. 581, 935-938.

Dean, M., Rzhetsky, A., and Allikmets, R. (2001). The human ATP-binding cassette $(\mathrm{ABC})$ transporter superfamily. Genome Res. 11, 1156-1166.

DeCarvalho, A. C., Gansheroff, L. J., and Teem, J. L. (2002). Mutations in the nucleotide binding domain 1 signature motif region rescue processing and functional defects of cystic fibrosis transmembrane conductance regulator delta f508. J. Biol. Chem. 277, 35896-35905.

Denning, G. M., Anderson, M. P., Amara, J. F., Marshall, J., Smith, A. E., and Welsh, M. J. (1992). Processing of mutant cystic fibrosis transmembrane conductance regulator is temperature-sensitive. Nature 358, 761-764.

Devidas, S., Yue, H., and Guggino, W. B. (1998). The second half of the cystic fibrosis transmembrane conductance regulator forms a functional chloride channel. J. Biol. Chem. 273, 29373-29380.

Di Bartolo, N. D., Hvorup, R. N., Locher, K. P., and Booth, P. J. (2011) In vitro folding and assembly of the Escherichia coli ATP-binding cassette transporter, BtuCD. J. Biol. Chem. 286, 18807-18815.

Du, K., and Lukacs, G. L. (2009). Cooperative assembly and misfolding of CFTR domains in vivo. Mol. Biol. Cell 20, 1903-1915.

Du, K., Sharma, M., and Lukacs, G. L. (2005). The deltaF508 cystic fibrosis mutation impairs domaindomain interactions and arrests post-translational folding of CFTR. Nat. Struct. Mol. Biol. 12, 17-25.

Gadsby, D. C. (2009). Ion channels versus ion pumps: the principal difference, in principle. Nat. Rev. Mol. Cell Biol. 10, 344-352.

Gadsby, D. C., Vergani, P., and Csanady, L. (2006). The ABC protein turned chloride channel whose failure causes cystic fibrosis. Nature 440 , 477-483.

Glozman, R., Okiyoneda, T., Mulvihill, C. M., Rini, J. M., Barriere, H., and Lukacs, G. L. (2009). N-glycans are direct determinants of CFTR folding and stability in secretory and endocytic membrane traffic. J. Cell Biol. $184,847-862$.

Gottesman, M. M., and Ambudkar, S. V. (2001). Overview: ABC transporters and human disease. J. Bioenerg. Biomembr. 33, 453-458.

Gregory, R. J., Rich, D. P., Cheng, S. H., Souza, D. W., Paul, S., Manavalan, P., Anderson, M. P., Welsh, M. J., and Smith, A. E. (1991). Maturation and function of cystic fibrosis transmembrane conductance regulator variants bearing mutations in putative nucleotide-binding domains 1 and 2. Mol. Cell. Biol. 11, 3886-3893.

Grove, D. E., Fan, C. Y., Ren, H. Y., and Cyr, D. M. (2011). The endoplasmic reticulum-associated Hsp40 DNAJB12 and Hsc70 cooperate to facilitate RMA1 E3dependent degradation of nascent CFTRdeltaF508. Mol. Biol. Cell 22, 301-314.

Grove, D. E., Rosser, M. F., Ren, H. Y., Naren, A. P., and Cyr, D. M. (2009). Mechanisms for rescue of correctable folding defects in
CFTRdelta F508. Mol. Biol. Cell 20, 4059-4069.

He, L., Aleksandrov, A. A., Serohijos, A. W., Hegedus, T., Aleksandrov, L. A., Cui, L., Dokholyan, N. V. and Riordan, J. R. (2008). Multiple membrane-cytoplasmic domain contacts in the cystic fibrosis transmembrane conductance regulator (CFTR) mediate regulation of channel gating. J. Biol. Chem. 283, 26383-26390.

Helenius, A., and Aebi, M. (2001). Intracellular functions of $\mathrm{N}$-linked glycans. Science 291, 2364-2369.

Hoelen, H., Kleizen, B., Schmidt, A. Richardson, J., Charitou, P., Thomas, P. J., and Brakkman, I. (2010). The primary folding defect and rescue of deltaF508 CFTR emerge during translation of the mutant domain. PLoS ONE 5, el5458. doi:10.1371/journal.pone.0015458

Hohl, M., Briand, C., Grutter, M. G., and Seeger, M. A. (2012). Crystal structure of a heterodimeric $\mathrm{ABC}$ transporter in its inward-facing conformation. Nat. Struct. Mol. Biol. 19, 395-402.

Holland, I. B. (2003). ABC Proteins: from Bacteria to Man. Amsterdam, Boston: Academic Press.

Hollenstein, K., Frei, D. C., and Locher, K. P. (2007). Structure of an $\mathrm{ABC}$ transporter in complex with its binding protein. Nature 446, 213-216.

Howard, M., Duvall, M. D., Devor, D. C., Dong, J. Y., Henze, K., and Frizzell, R. A. (1995). Epitope tagging permits cell surface detection of functional CFTR. Am. J. Physiol. 269, C1565-C1576.

Jensen, T. J., Loo, M. A., Pind, S., Williams, D. B., Goldberg, A. L., and Riordan, J. R. (1995). Multiple proteolytic systems, including the proteasome, contribute to CFTR processing. Cell 83, 129-135.

Jordan, I. K., Kota, K. C., Cui, G., Thompson, C. H., and McCarty, N. A. (2008). Evolutionary and functional divergence between the cystic fibrosis transmembrane conductance regulator and related ATP-binding cassette transporters. Proc. Natl. Acad. Sci. U.S.A. 105 18865-18870.

Kanelis, V., Hudson, R. P., Thibodeau, P. H., Thomas, P. J., and FormanKay, J. D. (2010). NMR evidence for differential phosphorylationdependent interactions in WT and deltaF508 CFTR. EMBO J. 29, 263-277.

Kerem, B., Rommens, J. M., Buchanan, J. A., Markiewicz, D., Cox, T. K., Chakravarti, A., Buchwald, M., and Tsui, L. C. (1989). Identification of the cystic fibrosis gene: genetic analysis. Science 245 , 1073-1080.

Kerem, E. (2004). Pharmacologic therapy for stop mutations: how much CFTR activity is enough? Curr. Opin. Pulm. Med. 10, 547-552.

Khushoo, A., Yang, Z., Johnson, A. E., and Skach, W. R. (2011). Ligand-driven vectorial folding of ribosome-bound human CFTR NBD1. Mol. Cell 41, 682-692.

Kleizen, B., Van Vlijmen, T., De Jonge, H. R., and Braakman, I. (2005). Folding of CFTR is predominantly cotranslational. Mol. Cell 20, 277-287.

Kramer, G., Ramachandiran, V., and Hardesty, B. (2001). Cotranslational folding-omnia mea mecum porto? Int. J. Biochem. Cell. Biol. 33, 541-553.

Lewis, H. A., Buchanan, S. G., Burley, S. K., Conners, K., Dickey, M., Dorwart, M., Fowler, R., Gao, X., Guggino, W. B., Hendrickson, W. A., Hunt, J. F., Kearins, M. C., Lorimer, D., Maloney, P. C., Post, K. W. Rajashankar, K. R., Rutter, M. E. Sauder, J. M., Shriver, S., Thibodeau, P. H., Thomas, P. J., Zhang, M., Zhao, $\mathrm{X}$., and Emtage, S. (2004). Structure of nucleotide-binding domain 1 of the cystic fibrosis transmembrane conductance regulator. EMBO J. 23 , 282-293.

Lewis, H. A., Wang, C., Zhao, X., Hamuro, Y., Conners, K., Kearins, M. C., Lu, F., Sauder, J. M., Molnar, K. S., Coales, S. J., Maloney, P. C., Guggino, W. B., Wetmore, D. R., Weber, P. C., and Hunt, J. F. (2010). Structure and dynamics of NBD1 from CFTR characterized using crystallography and hydrogen/deuterium exchange mass spectrometry. J. Mol. Biol. 396 406-430.

Lewis, H. A., Zhao, X., Wang, C., Sauder, J. M., Rooney, I., Noland, B. W., Lorimer, D., Kearins, M. C., Conners, K., Condon, B., Maloney, P. C., Guggino, W. B., Hunt, J. F., and Emtage, S. (2005). Impact of the deltaF508 mutation in first nucleotide-binding domain of human cystic fibrosis transmembrane conductance regulator on domain folding and structure. J. Biol. Chem. 280, 1346-1353.

Linsdell, P. (2006). Mechanism of chloride permeation in the cystic fibrosis transmembrane conductance regulator chloride channel. Exp. Physiol. 91, 123-129.

Linton, K. J., and Higgins, C. F. (1998). The Escherichia coli ATP-binding cassette (ABC) proteins. Mol. Microbiol. 28, 5-13. 
Locher, K. P. (2009). Review. Structure and mechanism of ATP-binding cassette transporters. Philos. Trans. R. Soc. Lond. B Biol. Sci. 364, 239-245.

Locher, K. P., Lee, A. T., and Rees, D. C. (2002). The E. coli BtuCD structure: a framework for $\mathrm{ABC}$ transporter architecture and mechanism. Science 296, 1091-1098.

Loo, T. W., Bartlett, M. C., and Clarke, D. M. (2011). Benzbromarone stabilizes deltaF508 CFTR at the cell surface. Biochemistry 50, 4393-4395.

Loo, T. W., and Clarke, D. M. (2008). Mutational analysis of $\mathrm{ABC}$ proteins. Arch. Biochem. Biophys. 476, 51-64.

Louie, R. J., Pagant, S., Youn, J. Y., Halliday, J. J., Huyer, G., Michaelis, S., and Miller, E. A. (2010). Functional rescue of a misfolded eukaryotic ATP-binding cassette transporter by domain replacement. J. Biol. Chem. 285, 36225-36234.

Lukacs, G. L., Chang, X. B., Bear, C., Kartner, N., Mohamed, A., Riordan, J. R., and Grinstein, S. (1993). The delta F508 mutation decreases the stability of cystic fibrosis transmembrane conductance regulator in the plasma membrane. Determination of functional half-lives on transfected cells. J. Biol. Chem. 268, 21592-21598.

Lukacs, G. L., Mohamed, A., Kartner, N., Chang, X. B., Riordan, J. R., and Grinstein, S. (1994). Conformational maturation of CFTR but not its mutant counterpart (delta F508) occurs in the endoplasmic reticulum and requires ATP. EMBO J. 13, 6076-6086.

Meacham, G. C., Lu, Z., King, S., Sorscher, E., Tousson, A., and Cyr, D. M. (1999). The Hdj-2/Hsc70 chaperone pair facilitates early steps in CFTR biogenesis. EMBO J. 18, 1492-1505.

Meacham, G. C., Patterson, C., Zhang, W., Younger, J. M., and Cyr, D. M. (2001). The Hsc70 co-chaperone CHIP targets immature CFTR for proteasomal degradation. Nat. Cell Biol. 3, 100-105.

Mendoza, J. L., Schmidt, A., Li, Q., Nuvaga, E., Barrett, T., Bridges, R. J., Feranchak, A. P., Brautigam, C. A., and Thomas, P. J. (2012). Requirements for efficient correction of deltaF508 CFTR revealed by analyses of evolved sequences. Cell 148, 164-174.

Mendoza, J. L., and Thomas, P. J. (2007). Building an understanding of cystic fibrosis on the foundation of $\mathrm{ABC}$ transporter structures. J. Bioenerg. Biomembr. 39, 499-505.
Mense, M., Vergani, P., White, D. M., Altberg, G., Nairn, A. C., and Gadsby, D. C. (2006). In vivo phosphorylation of CFTR promotes formation of a nucleotide-binding domain heterodimer. EMBO J. 25, 4728-4739.

Moody, J. E., Millen, L., Binns, D., Hunt, J. F., and Thomas, P. J. (2002). Cooperative, ATP-dependent association of the nucleotide binding cassettes during the catalytic cycle of ATPbinding cassette transporters. J. Biol. Chem. 277, 21111-21114.

Mornon, J. P., Lehn, P., and Callebaut, I. (2008). Atomic model of human cystic fibrosis transmembrane conductance regulator: membrane-spanning domains and coupling interfaces. Cell. Mol. Life Sci. 65, 2594-2612.

Mornon, J. P., Lehn, P., and Callebaut, I. (2009). Molecular models of the open and closed states of the whole human CFTR protein. Cell. Mol. Life Sci. 66, 3469-3486.

Muallem, D., and Vergani, P. (2009). Review. ATP hydrolysis-driven gating in cystic fibrosis transmembrane conductance regulator. Philos. Trans. R. Soc. Lond. B Biol. Sci. 364, 247-255.

Naren, A. P., Cormet-Boyaka, E., Fu, J., Villain, M., Blalock, J. E., Quick, M. W., and Kirk, K. L. (1999). CFTR chloride channel regulation by an interdomain interaction. Science 286, 544-548.

Nikles, D., and Tampe, R. (2007). Targeted degradation of $\mathrm{ABC}$ transporters in health and disease. $J$. Bioenerg. Biomembr. 39, 489-497.

Okiyoneda, T., Barriere, H., Bagdany, M., Rabeh, W. M., Du, K., Hohfeld J., Young, J. C., and Lukacs, G. L. (2010). Peripheral protein quality control removes unfolded CFTR from the plasma membrane. Science $329,805-810$.

Okiyoneda, T., and Lukacs, G. L. (2007). Cell surface dynamics of CFTR: the ins and outs. Biochim. Biophys. Acta 1773, 476-479.

Ostedgaard, L. S., Rich, D. P., Deberg, L. G., and Welsh, M. J. (1997). Association of domains within the cystic fibrosis transmembrane conductance regulator. Biochemistry 36, 1287-1294.

Pagant, S., Brovman, E. Y., Halliday, J. J., and Miller, E. A. (2008). Mapping of interdomain interfaces required for the functional architecture of Yorlp, a eukaryotic ATP-binding cassette (ABC) transporter. J. Biol. Chem. 283, 26444-26451.

Pagant, S., Halliday, J. J., Kougentakis, C., and Miller, E. A. (2010).
Intragenic suppressing mutations correct the folding and intracellular traffic of misfolded mutants of Yorlp, a eukaryotic drug transporter. J. Biol. Chem. 285 , 36304-36314.

Pagant, S., Kung, L., Dorrington, M., Lee, M. C., and Miller, E. A. (2007) Inhibiting endoplasmic reticulum (ER)-associated degradation of misfolded Yorlp does not permit ER export despite the presence of a diacidic sorting signal. Mol. Biol. Cell 18, 3398-3413.

Patrick, A. E., Karamyshev, A. L. Millen, L., and Thomas, P. J. (2011). Alteration of CFTR transmembrane span integration by diseasecausing mutations. Mol. Biol. Cell 22, 4461-4471.

Pind, S., Riordan, J. R., and Williams, D. B. (1994). Participation of the endoplasmic reticulum chaperone calnexin (p88, IP90) in the biogenesis of the cystic fibrosis transmembrane conductance regulator. J. Biol. Chem. 269, 12784-12788.

Pollet, J. F., Van Geffel, J., Van Stevens, E. Van Geffel, R., Beauwens, R., Bollen, A., and Jacobs, P. (2000). Expression and intracellular processing of chimeric and mutant CFTR molecules. Biochim. Biophys. Acta 1500 59-69.

Qu, B. H., Strickland, E. H., and Thomas, P. J. (1997). Localization and suppression of a kinetic defect in cystic fibrosis transmembrane conductance regulator folding. J. Biol. Chem. 272, 15739-15744.

Qu, B. H., and Thomas, P. J. (1996) Alteration of the cystic fibrosis transmembrane conductance regulator folding pathway. J. Biol. Chem. 271, 7261-7264.

Rabeh, W. M., Bossard, F., Xu, H. Okiyoneda, T., Bagdany, M., Mulvihill, C. M., Du, K., Di Bernardo, S., Liu, Y., Konermann, L., Roldan, A., and Lukacs, G. L. (2012). Correction of both NBD1 energetics and domain interface is required to restore deltaF508 CFTR folding and function. Cell 148 , 150-163.

Ramsey, B. W., Davies, J., McElvaney, N. G., Tullis, E., Bell, S. C., Drevinek, P., Griese, M., Mckone, E. F., Wainwright, C. E., Konstan, M. W., Moss R., Ratjen, F., Sermet-Gaudelus, I. Rowe, S. M., Dong, Q., Rodriguez, S., Yen, K., Ordonez, C., and Elborn, J. S. (2011). A CFTR potentiator in patients with cystic fibrosis and the G551D mutation. N. Engl. J. Med. 365, 1663-1672.

Rees, D. C., Johnson, E., and Lewinson, O. (2009). ABC transporters: the power to change. Nat. Rev. Mol. Cell Biol. 10, 218-227.

Rich, D. P., Gregory, R. J., Anderson, M. P., Manavalan, P., Smith, A. E., and Welsh, M. J. (1991). Effect of deleting the $\mathrm{R}$ domain on CFTRgenerated chloride channels. Science 253, 205-207.

Riordan, J. R. (2008). CFTR function and prospects for therapy. Annu. Rev. Biochem. 77, 701-726.

Riordan, J. R., Rommens, J. M., Kerem, B., Alon, N., Rozmahel, R., Grzelczak, Z., Zielenski, J., Lok, S., Plavsic, N., Chou, J. L., Drumm, M. L. Iannuzzi, M. C., Collin, F. S., and Tsui, L.-C. (1989). Identification of the cystic fibrosis gene: cloning and characterization of complementary DNA. Science 245, 1066-1073.

Rommens, J. M., Iannuzzi, M. C., Kerem, B., Drumm, M. L., Melmer, G., Dean, M., Rozmahel, R., Cole, J. L., Kennedy, D., Hidaka, N., Zsiga, M., Buchwald, M., Riordan, J. R., Tsui, L.-C., and Collins, F. S. (1989). Identification of the cystic fibrosis gene: chromosome walking and jumping. Science 245, 1059-1065.

Rosenberg, M. F., Kamis, A. B., Aleksandrov, L. A., Ford, R. C., and Riordan, J. R. (2004). Purification and crystallization of the cystic fibrosis transmembrane conductance regulator (CFTR). J. Biol. Chem. 279, 39051-39057.

Rosenberg, M. F., O'ryan, L. P., Hughes, G.,Zhao, Z., Aleksandrov, L. A., Riordan, J. R., and Ford, R. C. (2011). The cystic fibrosis transmembrane conductance regulator (CFTR): threedimensional structure and localization of a channel gate. J. Biol. Chem. 286, 42647-42654.

Rosser, M. F., Grove, D. E., Chen, L., and Cyr, D. M. (2008). Assembly and misassembly of cystic fibrosis transmembrane conductance regulator: folding defects caused by deletion of F508 occur before and after the calnexin-dependent association of membrane spanning domain (MSD) 1 and MSD2. Mol. Biol. Cell 19, 4570-4579.

Seibert, F. S., Linsdell, P., Loo, T. W., Hanrahan, J. W., Clarke, D M., and Riordan, J. R. (1996). Disease-associated mutations in the fourth cytoplasmic loop of cystic fibrosis transmembrane conductance regulator compromise biosynthetic processing and chloride channel activity. J. Biol. Chem. 271, 15139-15145.

Serohijos, A. W., Hegedus, T., Aleksandrov, A. A., He, L., Cui, L., Dokholyan, N. V., and Riordan, J. R. (2008). Phenylalanine-508 mediates 
a cytoplasmic-membrane domain contact in the CFTR 3D structure crucial to assembly and channel function. Proc. Natl. Acad. Sci. U.S.A. 105, 3256-3261.

Sheppard, D. N. (2011). Cystic fibrosis: CFTR correctors to the rescue. Chem. Biol. 18, 145-147.

Sheppard, D. N., Ostedgaard, L. S., Rich, D. P., and Welsh, M. J. (1994). The amino-terminal portion of CFTR forms a regulated Cl-channel. Cell 76, 1091-1098.

Smith, P. C., Karpowich, N., Millen, L., Moody, J. E., Rosen, J., Thomas, P. J., and Hunt, J. F. (2002). ATP binding to the motor domain from an $\mathrm{ABC}$ transporter drives formation of a nucleotide sandwich dimer. Mol. Cell 10, 139-149.

Strickland, E., Qu, B. H., Millen, L., and Thomas, P. J. (1997). The molecular chaperone $\mathrm{Hsc70}$ assists the in vitro folding of the $\mathrm{N}$-terminal nucleotide-binding domain of the cystic fibrosis transmembrane conductance regulator. J. Biol. Chem. 272, 25421-25424.

Sun, F., Zhang, R., Gong, X., Geng, X., Drain, P. F., and Frizzell, R. A. (2006). Derlin-1 promotes the efficient degradation of the cystic fibrosis transmembrane conductance regulator (CFTR) and CFTR folding mutants. J. Biol. Chem. 281, 36856-36863.

Teem, J. L., Berger, H. A., Ostedgaard, L. S., Rich, D. P., Tsui, L. C., and Welsh, M. J. (1993). Identification of revertants for the cystic fibrosis delta F508 mutation using STE6CFTR chimeras in yeast. Cell 73, 335-346.

Thibodeau, P. H., Brautigam, C. A., Machius, M., and Thomas, P. J. (2005). Side chain and backbone contributions of Phe508 to CFTR folding. Nat. Struct. Mol. Biol. 12, $10-16$
Thibodeau, P. H., Richardson, J. M. III, Wang, W., Millen, L., Watson, J., Mendoza, J. L., Du, K., Fischman, S., Senderowitz, H., Lukacs, G. L., Kirk, K., and Thomas, P. J. (2010). The cystic fibrosis-causing mutation deltaF508 affects multiple steps in cystic fibrosis transmembrane conductance regulator biogenesis. J. Biol. Chem. 285, 35825-35835.

Thomas, P. J., Shenbagamurthi, P., Sondek, J., Hullihen, J. M., and Pedersen, P. L. (1992). The cystic fibrosis transmembrane conductance regulator. Effects of the most common cystic fibrosis-causing mutation on the secondary structure and stability of a synthetic peptide. J. Biol. Chem. 267, 5727-5730.

Van Goor, F., Hadida, S., Grootenhuis, P. D., Burton, B., Cao, D., Neuberger, T., Turnbull, A., Singh, A., Joubran, J., Hazlewood, A., Zhou, J., McCartney, J., Arumugam, V., Decker, C., Yang, J., Young, C., Olson, E. R., Wine, J. J., Frizzell, R. A., Ashlock, M., and Negulescu, P. (2009). Rescue of CF airway epithelial cell function in vitro by a CFTR potentiator, VX770. Proc. Natl. Acad. Sci. U.S.A. 106, 18825-18830.

Vergani, P., Lockless, S. W., Nairn, A. C., and Gadsby, D. C. (2005). CFTR channel opening by ATP-driven tight dimerization of its nucleotidebinding domains. Nature 433, 876-880.

Wang, B., Heath-Engel, H., Zhang, D., Nguyen, N., Thomas, D. Y., Hanrahan, J. W., and Shore, G. C. (2008). BAP31 interacts with Sec61 translocons and promotes retrotranslocation of CFTRdeltaF508 via the derlin-1 complex. Cell 133, 1080-1092.

Wang, X., Venable, J., Lapointe, P., Hutt, D. M., Koulov, A. V., Coppinger, J., Gurkan, C., Kellner, W., Matteson,
J., Plutner, H., Riordan, J. R., Kelly, J. W., Yates, J. R. III, and Balch, W. E. (2006). Hsp90 cochaperone Ahal downregulation rescues misfolding of CFTR in cystic fibrosis. Cell 127 , 803-815.

Ward, A., Reyes, C. L., Yu, J., Roth, C. B., and Chang, G. (2007). Flexibility in the $\mathrm{ABC}$ transporter MsbA: alternating access with a twist. Proc. Natl. Acad. Sci. U.S.A. 104, 19005-19010.

Ward, C. L., Omura, S., and Kopito, R. R. (1995). Degradation of CFTR by the ubiquitin-proteasome pathway. Cell 83, 121-127.

Welsh, M. J., and Smith, A. E. (1993). Molecular mechanisms of CFTR chloride channel dysfunction in cystic fibrosis. Cell 73, 1251-1254.

Woolhead, C. A., McCormick, P. J. and Johnson, A. E. (2004). Nascent membrane and secretory proteins differ in FRET-detected folding far inside the ribosome and in their exposure to ribosomal proteins. Cell 116, 725-736

Xiong, X., Bragin, A., Widdicombe, J. H., Cohn, J., and Skach, W. R. (1997). Structural cues involved in endoplasmic reticulum degradation of G85E and G91R mutant cystic fibrosis transmembrane conductance regulator. J. Clin. Invest. 100, 1079-1088.

Younger, J. M., Chen, L., Ren, H. Y., Rosser, M. F., Turnbull, E. L., Fan, C. Y., Patterson, C., and Cyr, D. M. (2006). Sequential quality-control checkpoints triage misfolded cystic fibrosis transmembrane conductance regulator. Cell 126 571-582.

Zhang, F., Kartner, N., and Lukacs, G. L. (1998). Limited proteolysis as a probe for arrested conformational maturation of delta F508 CFTR. Nat. Struct. Biol. 5, 180-183.
Zhang, L., Aleksandrov, L. A., Riordan, J. R., and Ford, R. C. (2011). Domain location within the cystic fibrosis transmembrane conductance regulator protein investigated by electron microscopy and gold labelling. Biochim. Biophys. Acta 1808, 399-404.

Zhang, L., Aleksandrov, L. A., Zhao, Z., Birtley, J. R., Riordan, J. R., and Ford, R. C. (2009). Architecture of the cystic fibrosis transmembrane conductance regulator protein and structural changes associated with phosphorylation and nucleotide binding. J. Struct. Biol. 167, 242-251.

Zielenski, J., and Tsui, L. C. (1995). Cystic fibrosis: genotypic and phenotypic variations. Annu. Rev. Genet. 29, 777-807.

Conflict of Interest Statement: The authors declare that the research was conducted in the absence of any commercial or financial relationships that could be construed as a potential conflict of interest.

Received: 22 June 2012; paper pending published: 08 July 2012; accepted: 19 August 2012; published online: 06 September 2012.

Citation: Patrick $A E$ and Thomas PJ (2012) Development of CFTR structure. Front. Pharmacol. 3:162. doi 10.3389/fphar.2012.00162

This article was submitted to Frontiers in Pharmacology of Ion Channels and Channelopathies, a specialty of Frontiers in Pharmacology.

Copyright (c) 2012 Patrick and Thomas. This is an open-access article distributed under the terms of the Creative Commons Attribution License, which permits use, distribution and reproduction in other forums, provided the original authors and source are credited and subject to any copyright notices concerning any third-party graphics etc. 